\title{
Prevalence of Gingivitis Among Children Living in Bhopal
}

\section{Vinaykumar Hiremath', Nishi Mishra², Anandkumar G Patil ${ }^{3}$, Aparna Sheetal ${ }^{4}$, Sheetal Kumar}

\author{
ABSTRACT \\ Objective: Study was conducted to determine prevalence of gingivitis amongst school going children between 5-13 \\ years old around sub-urban area of Bhopal city.
}

Method: A total of 928 children (510 boys and 418 girls of age 5-13 years were examined). Out of 928 children 738 showed gingivitis.

Results: Prevalence of gingivitis was seen more in boys than girls. Age wise prevalence was evident amongst the children who were between the ages of 5-7 years.

Conclusion: This indicates the need for community health activities and awareness program.

Keywords: Gingivitis, Prevalence, Children

${ }^{1}$ Dean. Professor \& Head

Dept. of Oral and Maxillofacial Pathology Mansarovar Dental College Hospital and Research Centre, Kolar Road, Bhopal,

Madhya Pradesh, India.

2 Senior Lecturer

Dept. Oral Medicine and Radiology.

Mansarovar Dental College Hospital and

Research Centre, Kolar Road, Bhopal,

Madhya Pradesh, India.

${ }^{3}$ Professor \& Head

Dept. of Prosthodontics

Mansarovar Dental College Hospital and

Research Centre, Kolar Road, Bhopal,

Madhya Pradesh, India.

${ }^{4}$ Reader

Dept. of Pedodontics and Preventive Dentistry

Mansarovar Dental College Hospital and

Research Centre, Kolar Road, Bhopal,

Madhya Pradesh, India.

${ }^{5}$ Reader

Dept. of Oral and Maxillofacial Surgery

Mansarovar Dental College Hospital and

Research Centre, Kolar Road, Bhopal,

Madhya Pradesh, India.

\section{Contact Autbor}

Dr. Vinaykumar Hiremath hvinay20012001@yahoo.com

\section{INTRODUCTION}

$\longrightarrow$ ingivitis is one of the most com mon human diseases; Gingivitis of varying severity is nearly universal in children and adolescents. Gingivitis is characterized by the presence of gingival inflammation without detectable loss of bone. Redness, oedema and bleeding on probing characterize this condition. When treated gingivitis is reversible with no permanent damage. Untreated cases may lead to a more complex and destructive entity known as chronic periodontitis. Gingival diseases are broadly categorized into dental plaque induced and non plaque induced. Plaque induced gingival disease is the most common form of gingival diseases. It is caused by local factors and those affected by local factors modified by specific systemic factors of host. Bacteria and host interaction is modified by local and systemic factors. Local factors are micro-organisms, calculus, and food impacting faulty or irritating restorations, mouth breathing, and tooth mal-position (1).

Acute gingivitis is painful uncommon lesion with sudden onset and shorter duration. Gingivitis sometimes involves mar- ginal gingival known as Marginal gingivitis or inter-dental papilla, Papillary gingivitis, bleeding from the gingival sulcus following even mild irritation such as tooth brushing or probing is an early feature of gingivitis. Higher prevalence of gingivitis is reported in developing countries like India. Along with receiving professional care such as regular oral prophylaxis patients can choose various oral hygiene products for controlling plaque induced gingivitis. Good oral hygiene practices, including tooth-brushing and flossing using proper mouth rinses and receiving periodical oral prophylaxis can maintain gingival health.

Purpose of this study was to find the prevalence of gingivitis in school going children of Bhopal (M.P.)

\section{MATERIALS AND METHODS}

A sample size of 928 (510 males and 418 females) government school children were examined from Nov. 2010 to June 2011. Schools of Bhopal were randomly selected and with the consent of teachers and parents children were examined. Study was cleared by the institutional ethics commit- 
tee of Mansarovar Dental College, and children were referred to pediatric and preventive dentistry wing of Mansarovar Dental College. Children were examined by a single examiner who was trained to record the WHO (2) oral health assessment form to avoid inter-examiner variations. Loe and Silness (3) index was used for recording gingival status.

Loe-Silness gingival index:

$0=$ Absence of inflammation.

$1=$ Mild inflammation, slight change in color and texture.

2 = Moderate inflammation, moderate redness, edema, bleeding on probing, hypertrophy.

3 = severe inflammation: marked redness and hypertrophy, a tendency to spontaneous bleeding and ulceration.

Chi square test was applied and $\mathrm{P}$ value was taken out.

\section{RESULTS}

A total number of 928 children age between 5-13 years were examined out of which 510 were boys and 418 were girls, out of which 370 children belong to age group of 5-7 years, 282 children belong to age group of $8-10$ years and 276 belong to the age group of $11-13$ years. When age was taken into consideration $80 \%$ of the patients were having gingivitis in the age group of 5-7 years. $79 \%$ of the patients in the age group of $8-10$ years were having gingivitis. $78 \%$ of the patients in the age group of 11-13 years were having gingivitis; there is no significant relation between age group and gingivitis as $\mathrm{P}$ Value $>0.5$ (Table 1). When gender was taken into consideration out of 510 boys 418 (82\%) were having gingivitis and out of 418 girls 320 (76\%) were having gingivitis; there is significant relationship between gender and gingivitis as $\mathrm{P}$ Value $<0.5$ and accordingly

\section{Table 1: Relation Between Age Group And Gingivitis}

\begin{tabular}{|lcccccc|}
$\begin{array}{c}\text { Age in } \\
\text { years }\end{array}$ & $\begin{array}{c}\text { No. of } \\
\text { patients }\end{array}$ & Gingivitis & Healthy & $\begin{array}{c}\text { \% of Patients } \\
\text { with Gingivitis }\end{array}$ & Chi sq & P Value \\
\hline $5-7$ & 370 & 298 & 72 & 80.54 & 0.281 & 0.86892366 \\
$8-10$ & 282 & 224 & 58 & 79.43 & & \\
$11-13$ & 276 & 216 & 60 & 78.26 & & \\
\hline
\end{tabular}

Table 2: Relation Between Gender and Gingivitis

\begin{tabular}{|lcccccc|} 
Gender & $\begin{array}{l}\text { No. of } \\
\text { Patient }\end{array}$ & Gingivitis & Healthy & $\begin{array}{c}\text { \% of Patients } \\
\text { with Gingivitis }\end{array}$ & Chi. Sq. & P Value \\
\hline Male & 510 & 418 & 92 & 81.96 & & \\
Female & 418 & 320 & 98 & 76.56 & 4.123 & 0.042304 \\
\hline
\end{tabular}

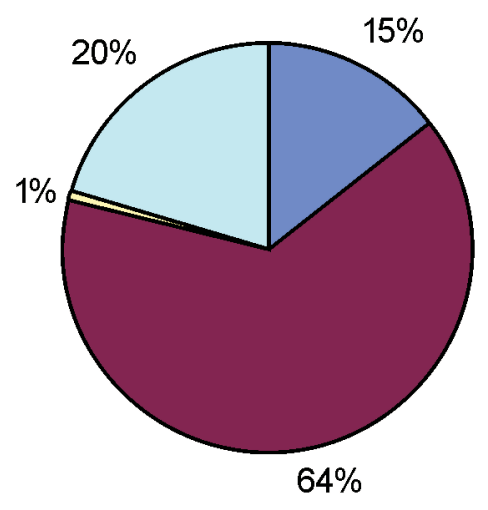

Mild Gingivitis

Moderate Gingivitis

Severe Gingivits

Healthy

Figure 1: Type of Gingivitis

percentage of gingivitis is higher in males (Table 2). When gingival index was considered $15 \%$ were having mild gingivitis, $64 \%$ of the patients were having moderate gingivitis, $1 \%$ were having severe gingivitis while $20 \%$ were found to be healthy. (Figure 1)

\section{DISCUSSION}

Studies on gingivitis have been conducted in many parts of the world on different ethical and cultural background, but periodic evaluation of data is very much required. Majority of the students examined in our study used tooth brush and paste to clean there teeth, some used finger or neem stick as a method of cleansing. When age wise prevalence was seen it was found $80 \%$ in $5-7$ years, $79 \%$ in $8-10$ years and $78 \%$ in $11-13$ years. When age was considered chi square value was 0.281 and $p$ value was $>0.87$ which is considered statistically insignificant. Decreased prevalence of gingivitis with increasing age may be attributed to the progressive accumulation of brushing knowledge by the children as they develop, which is contrary to the previous studies (4). When gender wise prevalence of gingivitis was considered boys were affected $82 \%$ and girls were affected $76 \%$ reason behind this can be attributed to the cleanliness of the girls. When gender was considered chi square value was 4.12 and $p$ value was $<0.042$ which is considered statistically significant. When gingival index was considered $80 \%$ of the children examined had gingivitis out of which $64 \%$ had moderate gingivitis, $15 \%$ had mild gingivitis and $1 \%$ had severe gingivitis while 20\% were found to be healthy. Previous studies shows contrast results in comparison to our results(5-7), reason behind may be the different type of socio economic conditions and geographical conditions.

There was no Periodontitis noted, results were concurrent with previous studies. It is a positive result that among young school children deeper tissue are not damaged only gingiva is affected for which treatment can be given. Presence of gingivitis in the school children can be due to presence of mixed dentition, varied food habits, improper and unsupervised oral hygiene practices, and malocclusion. 


\section{CONCLUSION}

Gingivitis progresses to periodontitis are the main cause of tooth loss. Prevalence of gingivitis needs an immediate attention in the children. There is a need to educate them about gingivitis; suitable oral health program using audio visual aid should be conducted. Prevalence and severity of gingivitis can be reduced by improving oral hygiene status.

\section{REFERENCE}

1. Shafer, Hine, Levy - Shafer's text book of oral pathology-2009, sixth edition

2. World Health Organization Oral Health Survey, Basic Methods, $4^{\text {th }}$ Edition; WHO: Geneva 1999.

3. Loe H, Silness J. Periodontal disease in pregnancy. I. Prevalence and severity Acta Odontological Scand 1963;21:53351.

4. Mahesh Kumar P, Joseph T, Varma RB, Jayanth M. Oral health status of 5 years and 12 years school going children in Chennai city. An epidemiological study. $J$
Indian Soc Pedo Prev Dent 2005;23:1722.

5. Jose A, Joseph MR. Prevalence of dental health problems among school going children in rural Kerala. J Indian Soc Pedo Prev Dent 2003;21:147-51.

6. Pandit K, Kannan AT, Sarana K, Agarwal K. Periodontal disease and dental caries in primary school children in rural areas of Delhi. Indian Journal of Pediatric 1986;53:525-29.

7. Mathur SK, Roy RK. Assessment of oral cleaning habit, bacterial plaque, gingivitis among school children. JIDA 1931;53: 329-32. 